Pacific Journal of Mathematics

LOCALLY GALOIS ALGEBRAS 


\title{
LOCALLY GALOIS ALGEBRAS
}

\author{
ANDY R. MAGID
}

\begin{abstract}
Separable subalgebras of commutative algebras which (a) are the direct limit of separable subalgebras and (b) have sufficiently many automorphisms are shown to be the fixed rings of groups of automorphisms of the algebra. Necessary and sufficient conditions for an arbitrary subalgebra to be the fixed ring of a group are examined.

Also, we show that every element of every separable algebra over a ring is separable if and only if the ring is von Neumann regular.
\end{abstract}

Given a commutative ring $R$ and a commutative $R$-algebra $S$, Villamayor and Zelinsky [10,3.1] call $S$ a weakly Galois $R$-algebra if $S$ is finitely generated and projective as an $R$-module, separable as an $R$-algebra, and if there is a finite group $G$ of $R$-algebra automorphisms of $S$ such that the subring $S^{G}$ of $G$-invariant elements of $S$ is precisely $R$. Under these hypotheses they achieve the following generalization of the fundamental theorem of Galois theory: Every separable $R$-subalgebra of $S$ is the fixed ring of a finite group of automorphisms, and conversely.

Our study here is of infinitely generated algebras. We say $S$ is a locally Galois $R$-algebra if every finite subset of $S$ is contained in a weakly Galois subalgebra of $S$ with the following property: there is a finite set of automorphisms of the subalgebra, each of which extends to an automorphism of $S$, and the fixed ring of the subalgebra under this subset is $R$. We prove that if $S$ is a locally Galois $R$ algebra, every separable $R$-subalgebra of $S$ is the fixed ring of some group of automorphisms of $S$. In general, the converse of this is false.

Call an $R$-algebra locally separable if every finite subset of the algebra is contained in a separable $R$-subalgebra. Under special hypotheses on $R$, which allow infinitely many idempotents, but are otherwise rather restrictive, we characterize those locally separable subalgebras of a locally Galois $R$-algebra which are the fixed rings of groups of automorphisms.

A major technique used here, as in [10], is to reduce to the case where $R$ has no nontrivial idempotents via Pierce's theory of the Boolean spectrum [7]: Let $X(R)$ denote the quotient space of Spec $(R)$ with connected components identified to points. The quotient map of Spec $(R)$ to $X(R)$ induces a sheaf on $X(R)$, the direct image of the canonical sheaf of local rings on $R$. The resulting ringed space 
$(X(R), \mathscr{F})$ is called the Boolean spectrum: the reason for the name and the fundamental properties of the Boolean spectrum are discussed in [7] and [10]. The principal feature is that each stalk of $\mathscr{F}$ has no nontrivial idempotents and that $(X(R), \mathscr{F})$ is universal with respect to this property.

o. Preliminaries. Unless explicitly noted to the contrary, all rings and algebras are assumed commutative with unit. $R$ denotes throughout the fixed, commutative base ring and all unadorned tensors are taken over $R$.

We shall use the following description of the Boolean spectrum $(X(R), \mathscr{F})$ of $R: X(R)$ is the maximal ideal space of the Boolean algebra $B(R)$ of idempotents of $R$, topologized by taking the sets $U_{e}=$ $\{x: 1-e \in x\}$, for all idempotents $e$, as basic open sets; and $\mathscr{F}\left(U_{e}\right)=$ Re. $U_{e} \subset U_{f}$ if and only if $e \leqq f$ (that is, $e f=e$ ). The stalk of $\mathscr{F}$ at $x$, which we denote $R_{x}$, is $R / x R$. The equivalence of this description and the one given in the introduction above is established in $[10,2.4]$.

(0.1) If $M$ is an $R$-module let $M_{x}=R \otimes R_{x}$ and for each $m$ in $M$ let $m_{x}$ be the image of $m$ in $M_{x}$.

(0.2) If $M$ and $N$ are $R$-modules and $f$ is in $\operatorname{Hom}_{R}(M, N)$, let $f_{x}$ be the morphism $f \otimes R_{x}$.

When $M$ is finitely presented the canonical map $\operatorname{Hom}_{R}(M, N)_{x} \rightarrow$ $\operatorname{Hom}_{R x}\left(M_{x}, N_{x}\right)$ is an isomorphism [3, p. 93, 2.8] and thus in this case the two interpretations of $f_{x}$ for $f$ in $\operatorname{Hom}_{R}(M, N)$ are compatible.

(0.3) An $R$-algebra $S$ is strongly separable if $S$ is a separable $R$-algebra which is finitely generated, projective and faithful as an $R$-module.

(0.4) A separable subalgebra of a strongly separable $R$-algebra is also strongly separable [6, p. 337, Remark 1].

For the convenience of the reader, we summarize here the results of [10] used in our study $\left(\operatorname{Aut}_{R}(S)\right.$ denotes the group of all $R$-algebra automorphisms of $S$ ):

(0.5) Suppose $R$ has no idempotents except 0 and 1 . If $S$ is a strongly separable $R$-algebra and if $S^{H}=R$ for some subgroup $H$ of $\operatorname{Aut}_{R}(S)$, then $H$ spans $\operatorname{Hom}_{R}(S, S)$ as a left $S$-module [10, 3.5]. 
(0.6) Let $S$ be an $R$-algebra and $H$ a subset of $\operatorname{Aut}_{R}(S)$. The closure of $H$ is the set of all automorphisms $g$ such that: for each $x$ in $X(R)$ and each minimal idempotent $f$ in $S_{x}, f \cdot g_{x}=f \cdot h_{x}$ for some $h$ in $H[10,3.7]$.

(0.7) Let $S$ be a weakly Galois $R$-algebra. If $F$ is a finite group of automorphisms of $S$ and if $T=S^{F}$, then $\operatorname{Aut}_{T}(S)$ is contained in the closure of $F$. [10,3.14]

(0.8) Let $S$ be a weakly Galois $R$-algebra, $T$ an $R$-subalgebra. Then $T$ is separable over $R$ if and only if $T=S^{F}$ for some finite subset $F$ of $\operatorname{Aut}_{R}(S)$. [10, 3.10 and 3.12]

For later use, we will need a stronger version of part of $(0.8)$. We remark that proof given here uses only [5] and (0.5) and hence is independent of $(0.8)$.

THEOREM 0.9. Let $S$ be a strongly separable $R$-algebra and $F$ a finite group of $R$-algebra automorphisms of $S$. Then $S^{F}$ is a strongly separable $R$-algebra.

Proof. Let $T=S^{F}$. Clearly $S$ is a separable $T$-algebra. We first assume that $R$ has no idempotents except 0 and 1 and show that (a) $S$ is $T$-projective and that (b) $S F=\operatorname{Hom}_{T}(S, S)$. Since $R$ has no nontrivial idempotents $S$ and hence $T$ have only finitely many idempotents. Let $e_{1}, \cdots, e_{n}$ be the minimal idempotents of $T$. To prove (a) and (b), we shall show that $S e_{i}$ is $T e_{i}$-projective and that

$$
S e_{i} F e_{i}=\operatorname{Hom}_{T e_{i}}\left(S e_{i}, S e_{i}\right)
$$

for $i=1, \cdots, n$; that is, we assume $T$ has no nontrivial idempotents. Let $e$ be a minimal idempotent of $S$ and let $H$ be the subgroup of $F$ fixing $e$. Since $T$ has no nontrivial idempotents, $F$ acts transitively on the minimal idempotents of $S$ and, exactly as in [9, p. 723, 1.3], we have that $T$ is isomorphic to $T e$ and $(S e)^{H}=T e$. By [5, p. 18, 1.3] $S e$ is $T$-projective. Thus $S$ is $T$-projective. Hence $S$ is weakly Galois over $T$ and, by (0.5), $S F=\operatorname{Hom}_{T}(S, S)$.

Now let $R$ be arbitrary. For each $x$ in $X(R)$,

$$
(S F)_{x}=S_{x} F_{x} \subseteq \operatorname{Hom}_{R x}\left(S_{x}, S_{x}\right) .
$$

By the above, $S_{x} F_{x}=\operatorname{Hom}_{S x} F_{x}\left(S_{x}, S_{x}\right)$, since $R_{x}$ has no nontrivial idempotents. Since also by the above $S_{x}$ is a projective $S_{x}^{F x}$-module, we see that $S_{x}^{F x}$ is separable over its center $S_{x} F_{x}$ [2, p. 380, 5.1] and that $S_{x}^{F x}$ is $R_{x}$-separable [1, p. 762, 4.8]. Thus $S_{x}^{F x}$ is $R_{x}$-separa- 
ble [2, p. 374, 2.5]. Since $S F$ is a finitely generated $R$-algebra we can now show that $S F$ is $R$-separable: for $S F$ is finitely presented as an $(S F)^{e}$-module, and thus it suffices [3, p. 94, 2.10] to show that for any maximal ideal $m$ of $R S F \otimes R_{m}$ is a separable $R_{m}$-algebra. Let $x$ be the set of idempotents in the kernel of $R \rightarrow R_{m}$. Since $R_{m}$ has no nontrivial idempotents, $x$ is in $X(R)$. Then

$$
S F \otimes R_{m}=\left(S F \otimes R_{x}\right) \otimes_{R x} R_{m}
$$

is $R_{m}$-separable. So the center $T$ of $S F$ is separable.

1. Separable subalgebras of locally Galois algebras. The principal result of this section is that separable subalgebras of locally Galois algebras are the fixed rings of groups of automorphisms. We begin by proving an extension theorem for some special subalgebras of arbitrary $R$-algebras; it is this theorem which motivates the definition 'locally Galois.' Our first lemma gives an equivalent definition of the closure (0.6) of a group of automorphisms.

Lemma 1.1. Let $S$ be an $R$-algebra finitely generated and projective as an $R$-module, and $H$ a subset of $\operatorname{Aut}_{R}(S)$. Then an automorphism $g$ is in the closure of $H$ if and only if there are pairwise orthogonal idempotents $e_{1}, \cdots, e_{n}$ of $S$ such that $\Sigma e_{j}=1$ and elements $h_{1}, \cdots, h_{n}$ of $H$, such that $g=\Sigma e_{j} h_{j}$.

Proof. Suppose that $g$ is in the closure of $H$. Let $x$ be in $X(R)$ and $f_{1}, \cdots, f_{k}$ be the minimal idempotents of $S_{x}$. By definition of the closure, there are $h_{1}, \cdots, h_{k}$ in $H$ such that

$$
f_{i} g_{x}=f_{i}\left(h_{i}\right)_{x}
$$

for $i=1, \cdots, k$. By [10, 2.12], choose idempotents $E_{1}, \cdots, E_{k}$ of $S$ such that $\left(E_{i}\right)_{x}=f_{i}$. These $E_{i}$ satisfy

$(*)$

$$
\begin{aligned}
\left(E_{i}\right)_{x}\left(E_{j}\right)_{x} & =\left(\delta_{i j} E_{i}\right)_{x} \\
\Sigma\left(E_{i}\right)_{x} & =1 \\
\left(E_{i} g\right)_{x} & =\left(E_{i} h_{i}\right)_{x} .
\end{aligned}
$$

By (0.1), there is a basic neighborhood $U_{e}$ of $x$ on which these equations are satisfied, i.e.,

$(* *)$

$$
\begin{aligned}
\left(e E_{i}\right)\left(e E_{j}\right) & =e\left(\delta_{i j} E_{i}\right) \\
e\left(\Sigma E_{i}\right) & =e \\
e\left(E_{i} g\right) & =e\left(E_{i} h\right) .
\end{aligned}
$$

For each $y$ in $X(R)$ there is a basic neighborhood of $y$ and a set of 
idempotents of $S$ and elements of $H$ satisfying $(*)$ on that neighborhood. By compactness, choose finitely many disjoint such neighborhoods covering $X(R)$; let these be $U_{e q}, q=1, \cdots, m$. Since the neighborhoods are disjoint, $e^{p} e^{q}=\delta_{p, q} e^{p}$ and since they cover $X(R), \Sigma e^{q}=1$. For each $q$ there is a set of idempotents of $S\left\{E_{i}^{q}\right\}$ and elements of $H\left\{h_{i}^{q}\right\}$ satisfying (**). The sets of idempotents $\left\{e^{q} E_{i}^{q}\right\}$ and elements of $H\left\{h_{i}^{q}\right\}$ then satisfy

$(* * *)$

$$
\begin{aligned}
\left(e^{q} E_{i}^{q}\right)\left(e^{p} E_{j}^{q}\right) & =\delta_{p, q} \delta_{i, j} e^{q} E_{i}^{q} \\
\Sigma e^{q} E_{i}^{q} & =1 \\
e^{q} E_{i}^{q} g & =e^{q} E_{i}^{q} h_{i}^{q} .
\end{aligned}
$$

Now, upon linearly ordering the indices $i$ and $q,(* * *)$ shows that there is a set $e_{1}, \cdots, e_{n}$ of pairwise orthogonal idempotents of $S$ such that $\Sigma e_{i}=1$ and elements $h_{1}, \cdots, h_{m}$ of $H$ such that $e_{i} g=e_{i} h_{i}, i=$ $1, \cdots, n$. Thus $g=\Sigma e_{i} h_{i}$.

If conversely $g=\Sigma e_{i} h_{i}$ where the $e_{i}$ and $h_{i}$ are as in the hypotheses of (1.1) then $e_{i} g=e_{i} h_{i}$ for $i=1, \cdots, n$ and $U e_{i}=1$. Hence by $[10,3.76], g$ is in the closure of $H$.

We remark that (1.1) improves [10, 3.76].

Lemma 1.2. Let $S$ be an $R$-algebra, $e_{1}, \cdots, e_{m}$ pairwise orthogonal idempotents of $S$ such that $\Sigma e_{i}=1$ and $h_{1}, \cdots, h_{m}$ elements of $\operatorname{Aut}_{R}(S)$. Suppose there is an $R$-subalgebra $T$ of $S$ such that each $e_{i}$ is in $T$, each $h_{i}$ leaves $T$ setwise invariant, and $g=\Sigma e_{i} h_{i}$ restricted to $T$ is in $\operatorname{Aut}_{R}(T)$. Then $g$ is in $\operatorname{Aut}_{R}(S)$.

Proof. Fix $j$ and choose $x$ in $T$ such that $g(x)=e_{j}$. Since

$$
\Sigma e_{i} h_{i}(x)=e_{j}, e_{j} h_{j}(x)=e_{j}
$$

and $e_{i} h_{i}(x)=0$ for $i \neq j$. Thus $h_{j}(x)=e_{j}+f$ where $e_{j}(f)=0$ and $x=h_{j}^{-1}\left(e_{j}\right)+h_{j}^{-1}(f)$. Then $h_{i}(x)=h_{i} h_{j}^{-1}\left(e_{j}\right)+h_{i} h_{j}^{-1}(f)$, for each $i$. If $i \neq j$, then $0=e_{i} h_{i}(x)=e_{i} h_{i} h_{j}^{-1}\left(e_{j}\right)+e_{i} h_{i} h_{j}^{-1}(f)$. This last expression is a sum of the form $a+b$, where $a$ is idempotent and $a b=0$; if such a sum is zero, $a$ must be zero. Hence $h_{i}\left(h_{i}^{-1}\left(e_{i}\right) h_{j}^{-1}\left(e_{j}\right)\right)=0$, so $h_{\imath}^{-1}\left(e_{i}\right) h_{\jmath}^{-1}\left(e_{j}\right)=0$. Thus the $h_{\imath}^{-1}\left(e_{i}\right)$ are pairwise orthogonal idempotents. Moreover, their sum is one: for the sum lies in $T$, and

$$
\left(\Sigma e_{i} h_{i}\right)\left(\Sigma h_{i}^{-1}\left(e_{i}\right)\right)=\Sigma e_{i} h_{i}\left(h_{\jmath}^{-1}\left(e_{j}\right)\right)=\Sigma \delta_{i j} e_{i}=1,
$$

and $g$ restricted to $T$ is an automorphism.

Now let $f_{i}=h_{i}^{-1}\left(e_{i}\right)$. If $s$ is any element of $S$, we have:

$$
\begin{aligned}
g\left(\Sigma f_{j} s\right) & =\Sigma g\left(f_{j} s\right)=\Sigma h_{i}\left(t_{i}\right) h_{i}\left(t_{j} s\right)=\Sigma h_{i}\left(f_{\imath} f_{j} s\right) \\
& =\Sigma h_{j}\left(f_{j} t_{j} s\right)=\Sigma e_{j} h_{j}\left(f_{j}\right) .
\end{aligned}
$$


Thus if we consider $g$ as a map of $\Pi S f_{i}$ to $\Pi S e_{i}$ we see that $g$ sends the factor $S f_{j}$ to the factor $S e_{j}$ by the algebra isomorphism $h_{j}$. Thus $g$ is an algebra automorphism of $S$.

THEOREM 1.3. Let $S$ be an R-algebra and $T$ a strongly separable subalgebra. Suppose there is a finite set $F$ of automorphisms of $T$ such that $T^{F}=R$ and each element of $F$ extends to an automorphism of $S$. Then every automorphism of $T$ extends to an automorphism of $S$.

Proof. Let $F_{0}$ be the subgroup of $\operatorname{Aut}_{R}(T)$ generated by $F$. [By $[10,3.14] F_{0}$ is finite.] Clearly each element of $F_{0}$ extends to an automorphism of $S$. Let $h$ be in $\operatorname{Aut}_{R}(T)$; by (0.7) $h$ is in the closure of $F_{0}$. By (1.1) $h=\Sigma e_{i} f_{i}$ where the $e_{i}$ are pairwise orthogonal idempotents of $T$ with $\Sigma e_{i}=1$ and each $f_{i}$ is in $F_{0}$. Extend each $f_{i}$ to an automorphism $g_{i}$ of $S$. By (1.2), $g=\Sigma e_{i} g_{i}$ is in $\operatorname{Aut}_{R}(S)$, and $g$ extends $h$.

Our definition of locally Galois algebra is essentially that every finite subset of the algebra belongs to a subalgebra to which (1.3) applies:

Definition 1.4. An $R$-algebra $S$ is called locally Galois if every finite subset of $S$ is contained in some strongly separable subalgebra $T$ with the following property: there is a finite subset $F$ of $\operatorname{Aut}_{R}(T)$ such that $T^{F}=R$ and each element of $F$ extends to an automorphism of $S$.

Lemma 1.5. Let $S$ be a locally Galois $R$-algebra and $T$ a strongly separable subalgebra. Then $T=S^{\mathrm{Aut}_{T}(S)}$.

Proof. Clearly $T$ is contained in $S^{\operatorname{aut} T(S)}$. Suppose $s$ is in $S \backslash T$ and choose a strongly separable subalgebra $W$ of $S$ containing $T$ and $s$ such that there is a finite subset $F$ of $\operatorname{Aut}_{R}(W)$ with $W^{F}=R$ and each element of $F$ extending to an automorphism of $S$. By (0.8), there is an $h$ in $\operatorname{Aut}_{R}(W)$ such that $h(s) \neq s$ but $h$ restricted to $T$ is the identity. Choose, by (1.3), an element $g$ of $\operatorname{Aut}_{R}(S)$ extending $h$. Then $g$ is in $\operatorname{Aut}_{T}(S)$ but $g(s) \neq s$. We conclude that $T=S^{\text {Aut }(S)}$.

THeOREM 1.6. Let $S$ be a locally Galois R-algebra and $T$ a separable subalgebra. Then $T$ is strongly separable and $T=S^{\text {AutT(S) }}$.

Proof. By (1.5), it suffices to show $T$ is strongly separable and by (0.4) it will suffice to show that $T$ is contained in a strongly 
separable $R$-algebra. Since $T$ is separable, there is an idempotent $e=\Sigma x_{i} \otimes y_{i}$ in $T \otimes T$ such that $\Sigma x_{i} y_{i}=1$ and for all $t$ in $T,(1 \otimes t) e=$ $(t \otimes 1) e$. We shall also use $e=\Sigma x_{i} \otimes y_{i}$ for the image of $e$ under $T \otimes T \rightarrow S \otimes S$. Choose a strongly subalgebra $W$ of $S$ containing all the $y_{i}$. For each $g$ in $\operatorname{Aut}_{W}(S)$, apply $1 \otimes g$ to the equation $(1 \otimes t) e=$ $(t \otimes 1) e$ and follow by the multiplication map $S \otimes S \rightarrow S$; we get

$$
g(t) \Sigma x_{i} y_{i}=t \Sigma x_{i} y_{i}
$$

since $g\left(y_{i}\right)=y_{i}$; since $\Sigma x_{i} y_{i}=1$ we get $g(t)=t$. Thus $T$ is contained in $S^{\mathrm{Aut} W(S)}$ and by (1.5) this latter is $W$.

We observe that the technique of (1.6) also gives the following:

Proposition 1.7. Let $S$ be an R-algebra and $C$ a class of subalgebras such that:

(a) Any finite subset of $S$ is contained in some element of $C$.

(b) For all $T$ in $C, T=S^{\text {4ut } T(S)}$.

Then every separable subalgebra of $S$ is contained in some element of $C$.

In light of (1.6), it is natural to try to describe all subalgebras of $S$ of the form $S^{H}$ for some subgroup $H$ of $\operatorname{Aut}_{R}(S)$. There are examples showing that such subalgebras need not even be a union of separable subalgebras. Something can be said, however, if $H$ is of a special type.

DEFSNITION 1.8. Let $S$ be an $R$-algebra and $H$ a group of $R$-algebra automorphisms of $S . H$ is almost finite if it has only finitely many restrictions to each finitely generated subalgebra of $S$.

Equivalent forms of (1.8) are given below in (1.12).

Proposition 1.9. Let $S$ be an $R$-algebra, $H$ an almost finite subgroup of $\operatorname{Aut}_{R}(S)$ and suppose that every finite subset of $S$ is contained in a strongly separable subalgebra of $S$. Then every finite subset of $S$ is contained in a strongly separable subalgebra left setwise invariant under $H$.

Proof. Let $F$ be a finite subset of $S$ and let $T_{0}$ be a strongly separable subalgebra containing $F$. Let $h_{1}, \cdots, h_{k}$ be the restrictions of $H$ to $T_{0}$ and let $T=h_{1}\left(T_{0}\right) \cdot h_{2}\left(T_{0}\right), \cdots, h_{k}\left(T_{0}\right)$. Clearly $T$ is left setwise invariant under $H . \quad T$ is an epimorphic image of $h_{1}\left(T_{0}\right) \otimes \ldots$ $\otimes h_{k}\left(T_{0}\right)$ hence finitely generated as an $R$-module and separable as an $R$-algebra. Under our hypotheses, $T$ is contained in a strongly separable subalgebra of $S$, hence by $(0.4)$ is itself strongly separable. 
THEOREM 1.10. Let $S$ be a locally Galois R-algebra, T, $T_{0}$ separable subalgebras of $S$ and $H$ an almost finite subgroup of $\operatorname{Aut}_{R}(S)$. Then:

(a) $T \cap T_{0}$ is strongly separable.

(b) $T \cap S^{I}$ is strongly separable.

(c) Every finite subset of $S^{H}$ is contained in a separable subalgebra of $S^{H}$.

Proof. (a) By (1.6), $T$ and $T_{0}$ are finitely generated. Thus, since $S$ is locally Galois, there is a strongly separable subalgebra $S_{0}$, containing both $T$ and $T_{0}$, for which there is a finite subset $F$ of $\operatorname{Aut}_{R}\left(S_{0}\right)$ with $S^{F}=R$. By $(0.8)$, then, there are finite subsets $K$ and $L$ of $\operatorname{Aut}_{R}\left(S_{0}\right)$ such that $T=S_{0}^{K}$ and $T_{0}=S_{0}^{L}$. We can assume, of course, that both $K$ and $L$ contain the identity. Then $T \cap T_{0}=S_{0}^{K L}$, where $K L$ is the set of products $k l$ for $k$ in $K, l$ in $L$. Thus by $(0.8)$ again, $T \cap T_{0}$ is strongly separable.

(b) Since by (1.6) $T$ is finitely generated, by (1.9) there is a strongly separable subalgebra $W$ of $S$ containing $T$ and left setwise invariant under $H$. By (1.6) also, $W=S^{J}$ where $J=\operatorname{Aut}_{W}(S)$. Since $W$ is setwise invariant under $H, H$ is contained in the normalizer of $J$ so $J$ is a normal subgroup of $J H$. Also, $W \cap S^{H}=\left(S^{J}\right)^{J H / J}$. Now $J H / J=H / J \cap H$ and this latter group is finite, since it consists of of the distinct restrictions of $H$ to $W$. By $(0.9)$, then, applied to the subalgebra $W \cap S^{H}$ of $W=S^{J}, W \cap S^{H}$ is $R$-separable. Now by (a), $T \cap S^{H}=T \cap\left(W \cap S^{H}\right)$ is also separable.

(c) Let $F$ be a finite subset of $S^{H}$. Choose a strongly separable subalgebra $V$ of $S$ containing $F$. Then by (b), $V \cap S^{H}$ is a separable subalgebra of $S^{H}$ containing $F$.

We formalize the property of $S^{H}$ established in (1.10) (c):

DeFinition 1.11. An $R$-algebra is said to be locally separable if every finite subset of the algebra is contained in a separable subalgebra.

There are examples showing that, without further hypotheses on $R$ it is neither necessary nor sufficient for a subalgebra of a locally Galois algebra to be locally separable to be the fixed ring of a group of automorphisms. Moreover, a locally separable subalgebra of a locally Galois algebra which is the fixed ring of a group need not be the fixed ring of an almost finite group.

The remainder of this section studies a special class of locally Galois algebras. We begin with equivalent characterizations of almost finite groups of automorphisms.

Proposition 1.12. Let $S$ be an $R$-algebra, $G$ a subgroup of $\operatorname{Aut}_{R}(S)$. 
The following are equivalent:

(a) $G$ is almost finite.

(b) For each $s$ in $S$ the orbit $G s$ is finite.

(c) For each $s$ in $S$ there is a normal subgroup $H$ of finite index in $G$ such that $s$ is in $S^{H}$.

(d) For any finite subset $F$ of $S$ there is a normal subgroup $H$ of finite index in $G$ such that $F$ is in $S^{H}$.

(e) $G$ is dense in a profinite group $G_{0}$ of algebra automorphisms of $S$ such that when $S$ carries the discrete topology, the action $G_{0} \times$ $S \rightarrow S$ is continuous.

These equivalences are well known, and we omit the standard proof.

We now cosider flat extensions of the base ring.

Lemma 1.13. Let $S, B, T$ be $R$-algebras, with $T$ a flat $R$-module, and $G$ an almost finite group of $R$-algebra automorphisms of $S$ such that $S^{G}=B$. Then $(S \otimes T)^{G \otimes 1}=B \otimes T$.

Proof. Let $x$ belong to $(S \otimes T)^{G \otimes 1}$, say $x=\Sigma s_{i} \otimes t_{i}$. Choose a normal subgroup $H$ of finite index in $G$ such that each $s_{i}$ is in $S^{H}$. Let $g_{1}, \cdots, g_{m}$ be coset representatives of $H$ in $G$. Then

$$
0 \longrightarrow B \longrightarrow S^{H} \longrightarrow \prod_{\imath=1}^{m} S^{H}
$$

is exact, where the last map sends $s$ to $\left(g_{1}(s)-s, \cdots, g_{m}(s)-s\right)$. Since by hypothesis $T$ is flat over $R$,

$$
0 \longrightarrow B \otimes T \longrightarrow S^{H} \otimes T \longrightarrow \Pi\left(S^{H} \otimes T\right)
$$

is exact, where $\left(\Pi S^{H}\right) \otimes T$ is identified with $\Pi\left(S^{H} \otimes T\right)$. The $j^{\text {th }}$ component of the last map of the sequence sends $y$ to $\left(g_{j} \otimes 1\right)(y)-y$ for any $y$ in $S^{H} \otimes T$. Thus since $x$ is in the kernel of this map, $x$ is in $B \otimes T$. Thus $(S \otimes T)^{G \otimes 1} \subseteq B \otimes T$; the reverse conclusion is obvious and the result.

Proposition 1.14. Let $S$ be an R-algebra such that:

(a) Every finite subset of $S$ is contained in a strongly separable subalgebra of $S$.

(b) There is an almost finite subgroup $G$ of $\operatorname{Aut}_{R}(S)$ such that $S^{R}=R$. Then $S$ is a locally Galois R-algebra.

Proof. By (1.9), every finite subset of $S$ is contained in a strongly separable subalgebra left setwise invariant under $G$. If $T$ is such a 
subalgebra, $F$ the group of restrictions of $G$ to $T$ then $F$ is finite, $T^{F}=R$, and each element of $F$ extends to an automorphism of $S$. Thus $S$ is locally Galois.

Proposition 1.15. Let $S$ be a locally Galois $R$-algebra and $T$ a flat $R$-algebra. The $S \otimes T$ is a locally Galois T-algebra. If $G$ is an almost finite group with $S^{G}=R, G \otimes 1$ is an almost finite group with $(S \otimes T)^{G \otimes 1}=T$.

Proof. The first assertion is obvious and the second is an immediate consequence of (1.13).

2. Locally separable subalgbras of locally Galois algebras. This section characterizes, under suitable hypotheses on the base ring $R$, those locally separable subalgebras of locally Galois algebras which are the fixed rings of automorphism groups. The concept of normality plays an important role in this.

Definition 2.1. Let $S$ be an $R$-algebra. An $R$-subalgebra $T$ of $S$ is said to be normal if every automorphism of $S$ sends $T$ into itself. $S$ is said to be a normally Galois $R$-algebra if every finite subset of $S$ is contained in a normal strongly separable subalgebra $T$ such that there is a finite subset $F$ of $\operatorname{Aut}_{R}(T)$ with $T^{F}=R$ and each element of $F$ extends to an automorphism of $S$. (Note that normally Galois implies locally Galois.)

Lemma 2.2. Let $S$ be an R-algebra and $T$ a subalgebra.

(a) If $T$ is normal, $\operatorname{Aut}_{T}(S)$ is a normal subgroup of $\operatorname{Aut}_{R}(S)$.

(b) If $H$ is a normal subgroup of $\operatorname{Aut}_{R}(S), S^{H}$ is a normal subalgebra.

Proposition 2.3. Let $S$ be a weakly Galois R-algebra, $T$ a normal, separable subalgebra and suppose $R$ is connected (i.e., has no nontrivial idempotents). Then either $T$ is connected or contains all idempotents of $S$.

Proof. Each element of $\operatorname{Aut}_{R}(S)$ restricted to the set $E$ of minimal idempotents of $S$ induces a permutation of $E$. If $E$ has $n$ elements, the restriction map $r$ induces a homomorphism of $\operatorname{Aut}_{R}(S)$ onto $S_{n}$, the symmetric group on $n$ symbols. $T=S^{\operatorname{AutT(S)}}$ and $\mathrm{Aut}_{T}(S)$ is normal by (2.2) (a). Thus $r\left(\operatorname{Aut}_{T}(S)\right)$ is a normal subgroup of $S_{n}$; if it is not the identity, it acts transitively on $E$. In the first case each element of $E$ is in $T$; in the second case, the only idempotents left fixed under $\operatorname{Aut}_{T}(S)$ are 0 and 1 , so $T$ is connected. 
Proposition 2.4. Let $R$ be connected and $S$ a locally Galois $R$ algebra. Then the following are equivalent:

(a) There is a collection $\mathscr{S}$ of weakly Galois subalgebras of $S$ such that every finite subset of $S$ is contained in some element of $\mathscr{S}$ and if $T_{1}, T_{2}$ are in $\mathscr{S}$ with $T_{1}$ contained in $T_{2}, T_{1}$ is normal in $T_{2}$.

(b) S has finitely many idempotents.

(c) $\operatorname{Aut}_{R}(S)$ is almost finite.

(d) $S$ is normally Galois.

Proof. $\quad(\mathrm{a}) \Rightarrow(\mathrm{b})$. Suppose $e$ is a nontrivial idempotent of $S$ and let $T$ be an element of $\mathscr{S}$ containing $e$. Let $f$ be any other idempotent of $S$ and $T_{1}$ an element of $\mathscr{S}$ containing both $T$ and $f . T$ is not connected and hence by (2.3) contains every idempotent of $T_{1}$, in particular $f$. Thus $T$ contains all idempotents of $S$; since $T$ is a finitely generated projective $R$-module these idempotents are finite in number.

(b) $\Rightarrow$ (c). Let $E=\left\{e_{1}, \cdots, e_{n}\right\}$ be the set of minimal idempotents of $S$ and $H$ the kernel of the restriction map of $\operatorname{Aut}_{R}(S)$ to the permutations of $S . H$ is the product of the groups $H_{i}=\operatorname{Aut}_{R e_{i}}\left(S e_{i}\right)$. Each $S e_{i}$ is connected and $\left(S e_{i}\right)^{H_{i}}=R e_{i}$. Since each $S e_{i}$ is locally separable, by $\left[6\right.$, p. 335, 1] each $H_{i}$ is almost finite. Then $H$ is almost finite and since $H$ is of finite index in $\operatorname{Aut}_{R}(S)$ this latter is almost finite.

(c) $\Rightarrow(\mathrm{d})$. Since $S^{\mathrm{Aut} R(S)}=R$, the implication follows from (1.9).

$(\mathrm{d}) \Rightarrow$ (a). Take for $\mathscr{S}$ the set of subalgebras satisfying the condition of $(2.1)$.

In $[9$, p. $723,1.3]$, it was shown that, over a connected ring, the full automorphism group of a weakly Galois algebra is finite.

EXAMPLE 2.5. The full automorphism group of a locally Galois algebra over a field need not be almost finite.

Let $R$ be a field and $S$ a Galois extension field of infinite degree over $R$. Clearly $S$ is a locally Galois $R$-algebra. By (1.15), $S \otimes S$ is a locally Galois $S$-algebra. Since $S$ is locally separable over $R, S$ is $S \otimes S$ flat so by [8, p. 94, 1] $S \otimes S$ is a von Neumann regular ring. If $\operatorname{Aut}_{S}(S \otimes S)$ is almost finite, by (2.4) $S \otimes S$ has only finitely many idempotents. A regular ring with only finitely many idempotents is a finite product of fields; it follows that the multiplication map $S \otimes$ $S \rightarrow S$ splits, so $S$ is $R$-separable. But since $S$ is $R$-projective, by [9, p. 722, 1.1] we also have $S$ finitely generated over $R$, contrary to hypothesis.

We remark that the Example (2.5) also shows that normally 
Galois is not preserved under flat base ring extension. In case the extension is by a stalk over a point in the Boolean spectrum, however, the property is stable.

Proposition 2.6. Let $S$ be a normally Galois $R$-algebra, $x$ a point in $X(R)$. Then $S_{x}$ is a normally Galois $R_{x}$-algebra.

Proof. By (1.15), $S_{x}$ is a locally Galois $R_{x}$-algebra. Let $\mathscr{S}$ be the collection of all normal subalgebras of $S$ satisfying the condition of (2.1). Let $\mathscr{S}_{x}=\left\{T_{x}: T \in \mathscr{S}\right\}$. Each element of $S_{x}$ is weakly Galois over $R_{x}$ and every finite subset of $S_{x}$ is contained in some element of $\mathscr{S}_{x}$. Let $T_{0}, T_{1}$ be elements of $\mathscr{S}_{x}$ with $T_{0} \subseteq T_{1}$ and let $T, T^{\prime}$ be elements of $\mathscr{S}$ with $T \subseteq T^{\prime}$ and $T_{x}=T_{0}, T_{x}^{\prime}=T_{1}$. Suppose $h$ is in $\operatorname{Aut}_{R x}\left(T_{1}\right)$. By $[10,2.14]$ choose $g$ in $\operatorname{Aut}_{R}\left(T^{\prime}\right)$ with $g_{x}=h$. Since $g(T) \subseteq T, h\left(T_{0}\right) \subseteq T_{0}$. Hence $T_{0}$ is a normal subalgebra $\mathrm{T}_{1}$. By (2.4) (a) $\Rightarrow$ (d), applied to $\mathscr{S}_{x}$, we conclude that $S_{x}$ is a normally Galois $R_{x}$ algebra.

Proposition 2.7. Let $R$ be connected, $S$ a normally Galois $R$ algebra and $T$ a locally separable subalgebra. Then $T=S^{H}$ where $H$ is the subgroup of $\operatorname{Aut}_{R}(S)$ fixing $T$.

Proof. Give $\operatorname{Hom}_{R}(S, S)$ the weakest topology such that for every finitely generated subalgebra $S_{0}$ of $S$ the restriction map $\operatorname{Hom}_{R}(S, S) \rightarrow$ $\operatorname{Hom}_{R}\left(S_{0}, S\right)$, induced by the inclusion of $S_{0}$ in $S$, is continuous. This is called the finite topology on $\operatorname{Hom}_{R}(S, S)$. We claim that $\operatorname{Aut}_{R}(S)$ is compact in this topology. For by $(2.4)(\mathrm{d}) \Rightarrow(\mathrm{c}), \operatorname{Aut}_{R}(S)$ is almost finite, and by (1.12) (a) $\Rightarrow(\mathrm{e}), \operatorname{Aut}_{R}(S)$ can be embedded in a compact group $G_{0}$ of automorphisms of $S$ such that the action $G_{0} \times S \rightarrow S$ is continuous. Thus $G_{0}=\operatorname{Aut}_{R}(S)$ is compact in the finite topology. Now the proof proceeds exactly as in the classical situation: let $s$ be in $S \backslash T$ and for each finitely generated separable subalgebra $T^{\prime}$ of $T$ let. $N\left(T^{\prime}\right)=\left\{g \in \operatorname{Aut}_{R}(S): g \mid T^{\prime}=i d\right.$ and $\left.g(s) \neq s\right\} . N\left(T^{\prime}\right)$ is a nonempty closed subset of $\operatorname{Aut}_{R}(S)$ and the collection of all $N\left(T^{\prime}\right)$, as $T^{\prime}$ ranges over the finitely generated separable subalgebras of $T$, has the finite intersection property. Then since $\operatorname{Aut}_{R}(S)$ is compact, there is a $g$ in $\operatorname{Aut}_{R}(S)$ which belongs to each $N\left(T^{\prime}\right)$. We have $g \mid T=i d$ and $g(s) \neq s$, and hence conclude that $T \supseteqq S^{H}$. Since the reverse inclusion is obvious, we have the result.

Now suppose that $R$ is arbitrary, $S$ is a normally Galois $R$-algebra, and $T$ is an almost separable subalgebra of $S$. Let $s$ be in $S \backslash T$ and $x$ a point in $X(R)$ such that $s_{x}$ is not in $T_{x}$. By (2.7), there is an $h$ in $\operatorname{Aut}_{R x}\left(S_{x}\right)$ such that $h \mid T_{x}=i d$ and $h\left(s_{x}\right) \neq s_{x}$. We would like to find a $g$ in $\operatorname{Aut}_{R}(S)$ such that $g \mid T=i d$ and $g_{x}=h_{x}$, so $g(s) \neq s$; that. 
is, we want to show first that $\operatorname{Aut}_{R}(S) \rightarrow \operatorname{Aut}_{R_{x}}\left(S_{x}\right)$ is onto and second that if $f$ and $g$ are in $\operatorname{Hom}_{R}(T, S)$ with $f_{x}=g_{x}$ in $\operatorname{Hom}_{R x}\left(T_{x}, S_{x}\right)$ (i.e., equal as maps) then $f=g$ on a neighborhood of $x$. In order to prove the second assertion, however, we will need to assume that $x$ is an isolated point of $X(R)$, and in this case the first assertion is trivial. For completeness, however, we include a more general result.

Lemma 2.8. Let $S$ be a normally Galois $R$-algebra, $G$ a profinite group such that $G \times S \rightarrow S$ is continuous and $S^{G}=R$, and $x$ a point in $X(R)$. Then $\operatorname{Aut}_{R}(S) \rightarrow \operatorname{Aut}_{R x}\left(S_{x}\right)$ is onto.

Proof. Let $\operatorname{Hom}_{R x}\left(S_{x}, S_{x}\right)$ carry the finite topology; note that $G_{x}$ is compact in this topology. Let $H=\operatorname{Aut}_{R x}\left(S_{x}\right) \cap\left(e_{1} G_{x}+\cdots+e_{n} G_{x}\right)$ where $e_{1}, \cdots, e_{n}$ are the minimal idempotents of $S$. $H$, being the intersection of compact sets, is closed in $\operatorname{Aut}_{R x}\left(S_{x}\right)$. We claim that $H$ is also dense in $\operatorname{Aut}_{R x}\left(S_{x}\right)$ : for the family of normal weakly Galois subalgebras of $S_{x}$ which contain all the $e_{i}, i=1, \cdots, n$, is cofinal, and hence to establish the claim it suffices to show that if $T$ is a subalgebra in the above mentioned cofinal family and $g$ in $\operatorname{Aut}_{R x}\left(S_{x}\right)$ then there is an $h$ in $H$ such that $g|T=h| T$. But this is precisely what was shown in (1.3). Hence $H=\operatorname{Aut}_{R x}\left(S_{x}\right)$.

Let $g$ be any element of $\operatorname{Aut}_{R x}\left(S_{x}\right)$; by the above, $g=e_{1} g_{1}+\cdots$ $+e_{n} g_{n}$, where $g_{i}$ is in $G_{x}$. Choose, by [10, 2.12], orthogonal idempotents $E_{1}, \cdots, E_{n}$ of $S$ such that $\left(E_{i}\right)_{x}=e_{i}$. Let $f$ be the sum of the $E_{i}$; we can assume $f$ is in $R$. Let $T$ be a normal weakly Galois subalgebra of $S f$ containing $E_{1}, \cdots, E_{n}$. Choose $h_{1}, \cdots, h_{n}$ in Gf such that $\left(h_{\imath}\right)_{x}=g_{i}$, and let $h=E_{1} g_{1}+\cdots+E_{n} g_{n}$. Since $(h \mid T)_{x}=g_{x} \mid T_{x}$ is an automorphism of $T_{x}$, there is an idempotent $e$ in $R f$ such that $h e \mid T e$ is an automorphism of Te. Hence by (1.2), he is an automorphism of $S e$. Then $h^{\prime}=(1-e)+h e$ is an automorphism of $S$ such that $h_{x}^{\prime}=g$.

One can show by example that, under the hypotheses of (2.8), $\operatorname{Aut}_{R}(S)_{x} \rightarrow \operatorname{Aut}_{R x}\left(S_{x}\right)$ need not be an isomorphism.

Lemma 2.9. Let $R$ be any commutative ring, $x$ an element of $X(R)$. Then $\operatorname{Hom}_{R}(M, N)_{x} \rightarrow \operatorname{Hom}_{R x}\left(M_{x}, N_{x}\right)$ is one to one for all $R$ modules $M$ and $N$ if and only if $R_{x}$ is $R$-projective.

Proof. Suppose $M$ is free, i.e., $M=\bigoplus R$. We have the following commutative diagram:

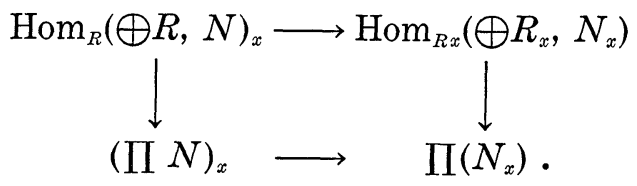


If the top map is one-to-one, so is the bottom, the vertical maps being isomorphisms. Now let $N=R$ and suppose the rank of $M$ is equal to the cardinality of $x$. Then if $\{f\}$ is a sequence in $\Pi R$ consisting of all the elements of $x$, we have $\{f\}_{x} \rightarrow\left\{f_{x}\right\}=\{0\}$. If the map is one-to-one, then, we have $\{f\}_{x}=0$; thus there is an idempotent $h$ of $R$ such that $(1-h) \in x$ and $h\{f\}=0$. Thus $(1-h) f=f$ for all $f$ in $x$, and $R x=R(1-h)$, so $R_{x}=R h$. Thus $R_{x}$ is $R$-projective.

If conversely $R_{x}$ is projective, we have $R_{x}=R e$ for some idempotent $e$, and if $M, N$ are $R$-modules, clearly

$$
\operatorname{Hom}_{R}(M, N) \otimes R e=\operatorname{Hom}_{R e}(M e, N e) .
$$

Lemma 2.10. Let $R$ be any commutative ring. Then $x$ in $X(R)$ is an isolated point if and only if $R x=R(1-f)$ for some (necessarily minimal) idempotent $f$ of $R$.

Proof. If $x$ is isolated, then $x=U_{f}$ for some idempotent $f$. Then $1-e$ is in $x$ if and only if $U_{f} \subseteq U_{e}$, i.e., if and only if $f e=f$, so $(1-f)(1-e)=1-e$. Thus $R x=R(1-f)$. Now suppose, conversely, that $R x=R(1-f)$ and $y$ is any element of $U_{f}$. Then $1-f \in y$, so $R x \leqq R y$. Let $P$ be a prime ideal of $R$ containing $R y ; P \cap B(R)$ is a maximal ideal of $B(R)$ containing both $x$ and $y$, hence equal to both $x$ and $y$. It follows that $U_{f}=\{x\}$, and thus $x$ is isolated.

We remark that by (2.9) and (2.10), if $x$ is an isolated point of $X(R)$ then both the properties discussed immediately before (2.8) hold for $x$ : by $(2.10), R_{x}=R f$, and hence

$$
\operatorname{Aut}_{R}(S)=\operatorname{Aut}_{R x}\left(S_{x}\right) \times \operatorname{Aut}_{R(1-f)}(S(1-f)) .
$$

Also, we many view (2.9) and (2.10) as evidence that the second property will not hold unless $x$ is isolated.

Definition 2.11. Let $S$ be a commutative $R$-algebra. A subalgebra $T$ of $S$ is called separated if given $s$ in $S$ but not in $T$ there is a minimal idempotent $e$ of $R$ such that $s e$ in not in $T e$.

THeOREm 2.12. Let $S$ be a normally Galois R-algebra, $T$ a separated locally separable subalgebra. Then $T=S^{\mathrm{AutT}(S)}$.

Proof. We need to show that if $s$ is an element of $S \backslash T$ there is a $g$ in $\operatorname{Aut}_{R}(S)$ such that $g(s) \neq s$ but $g \mid T=i d$. Since $T$ is separated, there is an isolated point $x$ of $X(R)$ such that $s_{x}$ is not in $T_{x}$. By (2.7), there is an $h$ in $\operatorname{Aut}_{R x}\left(S_{x}\right)$ such that $h \mid T_{x}=i d$ and $h\left(s_{x}\right) \neq s_{x}$. Since $x$ is isolated, there is a $g$ in $\operatorname{Aut}_{R}(S)$ with $g_{x}=h$ (as maps). 
Considering $g \mid T$, then, as an element of $\operatorname{Hom}_{R}(T, S)$ we have, by (2.9), that $(g \mid T)_{x}=g_{x} \mid T_{x}=i d_{x}$. By (2.10), there is a minimal idempotent $f$ of $R$ such that $R_{x}=R f$; hence $g f \mid T f=i d$. Replacing $g$ by ( $1-$ $f)+g f$ then gives the desired automorphism.

Theorem (2.12) gives a sufficient condition for a locally separable subalgebra of a normally Galois $R$-algebra to be the fixed ring of a subgroup of $\operatorname{Aut}_{R}(S)$. We now consider hypotheses on $R$ under which the condition becomes sufficient.

Recall that a ring is said to be semi-hereditary if every finitely generated submodule of a free module is projective. A ring is semihereditary if and only if every finitely generated ideal is projective, and hence a von Neumann regular ring is semi-hereditary.

THEOREM 2.13. Let $R$ be a semi-hereditary ring and suppose that every idempotent of $R$ contains a minimal idempotent. Let $S$ be a normally Galois R-algebra and $T$ a locally separable subalgebra of $S$. Then $T$ is the fixed ring of a subgroup of $\operatorname{Aut}_{R}(S)$ if and only if $T$ is separated.

Proof. By (2.12), we need only show that if $T=S^{H}$ for some subgroup $H$ of $\operatorname{Aut}_{R}(S)$ then $T$ is separated. Let $s$ be an element of $S$ and $T_{0}$ a normal weakly Galois subalgebra of $S$ containing $s$. Let $g$ be in $H$ and consider the $R$-submodule $R(g(s)-s)$ of $T_{0}$; since $R$ is semi-hereditary, this submodule is projective. For any $x$ in $X(R)$, $(R(g(s)-s))_{x}=R_{x}\left(g_{x}\left(s_{x}\right)-s_{x}\right)$. Thus if $g_{x}\left(s_{x}\right)=s_{x}$ for all $x$ in a dense subset of $X(R), R(g(s)-s)$ would be a projective $R$-module of rank zero on a dense subset of $X(R)$. Then since the rank function of a projective module is continuous on $X(R), R(g(s)-s)=0$ and $g(s)=s$.

The hypothesis on the idempotents of $R$ means that the isolated points of $X(R)$ are dense. Thus if $s$ is not in $T$, there is a $g$ in $H$ with $g(s) \neq s$ and hence, by the above, an isolated point $x$ of $X(R)$ such that $g_{x}\left(s_{x}\right) \neq s_{x}$. If $e$ is the minimal idempotent of $R$ such that $R_{x}=R e, T e=\left(S^{H}\right) e=(S e)^{H e}=S_{x}^{H x}$, so $s_{x}=s e$ is not in $T e$. It follows that $T$ is separated.

The hypothesis that $R$ be semi-hereditary was employed only to show that if $g_{x}\left(s_{x}\right)=s_{x}$ on a dense subset of $X(R), g(s)=s$. A similar result holds in general without hypotheses on $R$ if we assume that $s$ is a separable element.

Proposition 2.14. Let $S$ be a normally Galois R-algebra, $g$ an element of $\mathrm{Aut}_{R}(S)$ and $s$ a separable element of $S$ (i.e., $R[s]$ is a separable $R$-algebra). Then $g(s)=s$ if and only if $g_{x}\left(s_{x}\right)=s_{x}$ for all $x$ on a dense subset of $X(R)$. 
Proof. Let $T_{0}$ be a normal weakly Galois subalgebra containing $s$. Let $H$ be the subgroup of $\operatorname{Aut}_{R}\left(T_{0}\right)$ generated by $g \mid T_{0}$, and let $T=T_{0}^{H} . \quad H$ is finite $[10,2.16]$ and hence $T$ is a strongly separable $R$-algebra. Let $T^{\prime}=T[s] . \quad T^{\prime}$ is an epimorphic image of $T \otimes R[s]$, hence separable, and $T^{\prime}$ is contained in $T_{0}$, hence is strongly separable. Since $T \subseteq T^{\prime}, T$ is a direct summand of $T^{\prime}$ and $M=T^{\prime} / T$ is a finitely generated projective $R$-module. For each $x$ in $X(R), T_{x}=\left(T_{0}\right)_{x}^{H x}, T_{x}^{\prime}=$ $T_{x}\left[s_{x}\right]$ and $M_{x}=T_{x}^{\prime} / T_{x}$. Thus $g_{x}\left(s_{x}\right)=s_{x}$ if and only if $M_{x}=0$. Thus if $g_{x}\left(s_{x}\right)=s_{x}$ on a dense subset of $X(R), M_{x}$ has zero rank on a dense subset of $X(R)$; hence $M=0, T^{\prime}=T$ and $g(s)=s$. The converse is trivial.

With (2.14) available, it is clear that (2.13) can be proved with the hypothesis of semi-hereditary replaced by the condition that any element of any strongly separable $R$-algebra is a separable element. In fact, however, this condition on $R$ is much stronger than semihereditary. We shall show that it holds if and only if $R$ is a von Neumann regular ring.

Proposition 2.15. Let $R$ be a von Neumann regular ring, $S$ a strongly separable $R$-algebra. Then every element of $s$ is separable.

Proof. Let $s$ be in $S . \quad R[s]$ is finitely generated as an $R$-algebra, hence is separable if for each prime $p$ of $R(R[s])_{P}$ is separable over $R_{p}$. But since $R$ is regular, $\operatorname{Spec}(R)=X(R)$ and hence we need to show that each $(R[s])_{x}$ is separable to conclude the result. We have $(R[s])_{x}=R_{x}\left[s_{x}\right] \leqq S_{x}$, and hence, since $R_{x}$ is a field and $S_{x}$ is $R_{x}$-separable, $R[s]_{x}$ is $R_{x}$-separable.

LEMMA 2.16. Let $R$ be a connected ring and $S$ a strongly separable $R$ algebra not equal to $R$. If every element of $S$ is separable, every nonunit of $R$ is nilpotent.

Proof. First observe that every subalgebra of $S$ is separable: for by hypothesis every finitely generated subalgebra is separable, and hence every subalgebra is locally separable. Let $T$ be a subalgebra of $S$, and write $T$ as the union of an ascending chain $\left\{T_{i}\right\}$ of separable subalgebras. Since $S$ is strongly separable, each of these subalgebras is a direct summand of $S$. It follows that the ranks of the $S / T_{i}$ must properly decrease as $i$ increases, and hence is constant for $i \geqq j$, some $j$. But then since $T_{j}$ is a summand of $T_{i}$ for all $i \geqq j$, and $T_{i}$ and $T_{j}$ have the same rank, $T_{i}=T_{j}$ for all $i \geqq j$.

Now let $b$ be a nonunit of $R$ which is not nilpotent. Let $m$ be a maximal ideal of $R$ containing $b$. We consider the subalgebra $T=$ $R+m S$ of $S$. We will show that $T \neq R$ and $T \neq S$. Thus $T$ is a 
strongly separable $R$-algebra, not equal to $R$, such that every element of $T$ is $R$-separable, and $[T: R]<[S: R]$. By induction, then, we have a contradiction. If $T=S, S / m S=R+m S / m S=R / R \cap m S$ is of rank $\leqq 1$ over $R / m$. This is impossible since $S \neq R$ and hence $[S: R]>1$. If $T=R, S b \leqq R$ so $b$ annihilates $S / R$, which is a finitely generated projective $R$-module of rank $\geqq 1$. Let $p$ be a prime of $R$. If $b$ is not in $p, S_{p} / R_{p}$ is zero since it is annihilated by a unit of $R_{p}$; on the other hand $\left[S_{p} / R_{p}: R_{p}\right] \geqq 1$. Hence $b$ must be in $p$. But then $b$ is in every prime of $R$, hence nilpotent, contrary to assumption.

Proposition 2.17. If every element of every strongly separable $R$-algebra is separable, then $R$ is a von Neumann regular ring.

Proof. Let $x$ be a point of $X(R)$; since $R \times R$ is a strongly separable $R$-algebra, every element of $R_{x} \times R_{x}$ is separable over $R_{x}$. Thus, by (2.16) every nonunit of $R_{x}$ is nilpotent. Let $r$ be in $R_{x}$ with $r^{2}=0$. If $r \neq 0, R_{x}[(r, 0)]$ is a separable subalgebra of $R_{x} \times R_{x}$ not equal to $R_{x}$, hence of rank $\geqq 2$. Thus $R_{x}[(r, 0)]=R_{x} \times R_{x}$. But every element of $R_{x}[(r, 0)]$ may be written as $t(1,1)+s(r, 0)=(t+s r, t)$ for some $s, t$ in $R_{x}$. Hence there are $s, t$ such that $(t+s r, t)=(0,1)$, i.e., $1=-s r$. Thus $r$ is a unit, contradiction. It follows that $R_{x}$ has no nilpotents, hence is a field, and since each $R_{x}$ is a field, $R$ is von Neumann regular.

This paper is based on the author's doctoral dissertation (Northwestern, 1969). Thanks are due to Professor Daniel Zelinsky under whose guidance this thesis was written.

\section{REFERENCES}

1. M. Auslander and D. Bucksbaum, On ramification theory in Noetherian rings, Amer. J. Math. 81 (1959), 749-765.

2. M. Auslander and O. Goldman, The Brauer group of a commutative ring, Trans. Amer. Math. Soc. 97 (1960), 367-409.

3. H. Bass, Lectures on topics in algebraic K-theory, Tata Institute of Fundamental Research, Bombay, 1967.

4. N. Bourbaki, Algebre commutative, Chapitres 5 et 6, Hermann, Paris, 1964.

5. S. Chase, D. Harrison, and A. Rosenberg, Galois theory and Galois cohomology of commutative rings, Memoirs Amer. Math. Soc. 521965.

6. T. Nagahara, $A$ note on Galois theory of commutative rings, Proc. Amer. Math. Soc. 18 (1967), 334-340.

7. R. Pierce, Modules over commutative regular rings, Memoirs Amer. Math. Soc. 70 1967.

8. O. Villamayor, On weak dimension of algebras, Pacific J. Math. 9 (1959) 943-951. 
9. O. Villamayor and D. Zelinsky, Galois theory for rings with finitely many idempotents, Nagoya Math. J. 27 (1966), 721-731.

10. — Galois theory for rings with infinitely many idempotents, Nagoya Math. J. 35 (1969), 83-98.

Received August 11, 1969.

NORTHWESTERN UNIVERSITY

AND

Columbia UNIVERSity 


\section{PACIFIC JOURNAL OF MATHEMATICS}

\section{EDITORS}

\author{
H. SAMELSON \\ Stanford University \\ Stanford, California 94305 \\ Richard Pierce \\ University of Washington \\ Seattle, Washington 98105
}

J. DUGUNDJI

Department of Mathematics

University of Southern California

Los Angeles, California 90007

RICHARD ARENS

University of California

Los Angeles, California 90024

\section{ASSOCIATE EDITORS}
E. F. BECKENBACH
B. H. NEUMaNN
F. WOLE
K. YosHIDA

\section{SUPPORTING INSTITUTIONS}

\author{
UNIVERSITY OF BRITISH COLUMBIA \\ CALIFORNIA INSTITUTE OF TECHNOLOGY \\ UNIVERSITY OF CALIFORNIA \\ MONTANA STATE UNIVERSITY \\ UNIVERSITY OF NEVADA \\ NEW MEXICO STATE UNIVERSITY \\ OREGON STATE UNIVERSITY \\ UNIVERSITY OF OREGON \\ OSAKA UNIVERSITY \\ UNIVERSITY OF SOUTHERN CALIFORNIA
}

\author{
STANFORD UNIVERSITY \\ UNIVERSITY OF TOKYO \\ UNIVERSITY OF UTAH \\ WASHINGTON STATE UNIVERSITY \\ UNIVERSITY OF WASHINGTON

$* * *{ }^{*} *{ }^{*}$
AMERICAN MATHEMATICAL SOCIETY
CHEVRON RESEARCH CORPORATION
TRW SYSTEMS \\ NAVAL WEAPONS CENTER
}

The Supporting Institutions listed above contribute to the cost of publication of this Journal. but they are not owners or publishers and have no responsibility for its content or policies.

Mathematical papers intended for publication in the Pacific Journal of Mathematics should be in typed form or offset-reproduced, (not dittoed), double spaced with large margins. Underline Greek letters in red, German in green, and script in blue. The first paragraph or two must be capable of being used separately as a synopsis of the entire paper. The editorial "we" must not be used in the synopsis, and items of the bibliography should not be cited there unless absolutely necessary, in which case they must be identified by author and Journal, rather than by item number. Manuscripts, in duplicate if possible, may be sent to any one of the four editors. Please classify according to the scheme of Math. Rev. 36, 1539-1546. All other communications to the editors should be addressed to the managing editor, Richard Arens, University of California, Los Angeles, California, 90024.

50 reprints are provided free for each article; additional copies may be obtained at cost in multiples of 50 .

The Pacific Journal of Mathematics is published monthly. Effective with Volume 16 the price per volume (3 numbers) is $\$ 8.00$; single issues, $\$ 3.00$. Special price for current issues to individual faculty members of supporting institutions and to individual members of the American Mathematical Society: $\$ 4.00$ per volume; single issues $\$ 1.50$. Back numbers are available.

Subscriptions, orders for back numbers, and changes of address should be sent to Pacific Journal of Mathematics, 103 Highland Boulevard, Berkeley, California, 94708.

PUBLISHED BY PACIFIC JOURNAL OF MATHEMATICS, A NON-PROFIT CORPORATION

Printed at Kokusai Bunken Insatsusha (Internatıonal Academic Printing Co., Ltd.), 7-17, Fujimi 2-chome, Chiyoda-ku, Tokyo, Japan. 


\section{Pacific Journal of Mathematics}

\section{Vol. 33, No. 3 \\ May, 1970}

Charles A. Akemann, Approximate units and maximal abelian

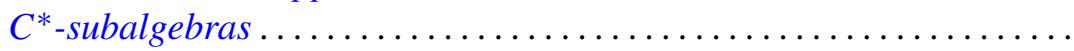

Gail Atneosen, Wild points of cellular arcs in 2-complexes in $E^{3}$ and cellular hulls

John Logan Bryant and De Witt Sumners, On embeddings of 1-dimensional

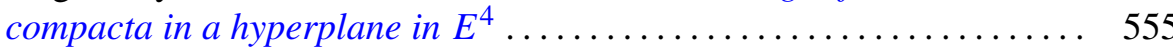

H. P. Dikshit, On a class of Nörlund means and Fourier series.......... 559

Nancy Dykes, Generalizations of realcompact spaces .............. 571

Hector O. Fattorini, Extension and behavior at infinity of solutions of certain linear operational differential equations .................. 583

Neal David Glassman, Cohomology of nonassociative algebras .......... 617

Neal Hart, Ulm's theorem for Abelian groups modulo bounded groups . . . . 635

Don Barker Hinton, Continuous spectra of second-order differential

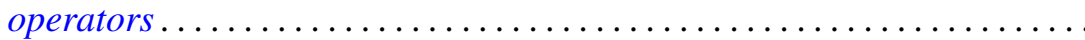

Donald Gordon James, On Witt's theorem for unimodular quadratic forms. II ............................................. 645

Melvin F. Janowitz, Principal multiplicative lattices................ 653

James Edgar Keesling, On the equivalence of normality and compactness in hyperspaces .................................... 657

Adalbert Kerber, Zu einer Arbeit von J. L. Berggren über ambivalente Gruppen

Keizō Kikuchi, Various $m$-representative domains in several complex variables

Jack W. Macki and James Stephen Muldowney, The asymptotic behaviour of solutions to linear systems of ordinary differential equations ....

Andy R. Magid, Locally Galois algebras . . . .

T. S. Ravisankar, On differentiably simple algebras......

Joseph Gail Stampfli, The norm of a derivation ..

Francis C.Y. Tang, On uniqueness of central decompositions of groups ...

749

Robert Charles Thompson, Some matrix factorization theorems. I .

763

Robert Charles Thompson, Some matrix factorization theorems. II . . 